\title{
Gliquidone decreases urinary protein by promoting tubular reabsorption in diabetic Goto-Kakizaki rats
}

\author{
Jian-Ting Ke*, Mi Li", Shi-Qing Xu', Wen-Jian Zhang', Yong-Wei Jiang', \\ Lan-yun Cheng', Li Chen ${ }^{2}$, Jin-Ning Lou' and Wei Wu \\ Department of Nephrology, Fifth Affiliated Hospital, Sun Yat-Sen University, Zhuhai 519000, \\ People's Republic of China \\ ${ }^{1}$ Institute of Clinical Medical Sciences, China-Japan Friendship Hospital, Beijing 100029, People's Republic of China \\ ${ }^{2}$ Department of Endocrinology, Qilu Hospital, Shandong University, Jinan 250012, Shandong, China \\ *(J-T Ke and M Li contributed equally to this work)
}

\author{
Correspondence \\ should be addressed \\ to J-N Lou or W Wu \\ Emails \\ Lou.j@mail.com or \\ wu.wei@medmail.com
}

\begin{abstract}
The efficacy of gliquidone for the treatment of diabetic nephropathy was investigated by implanting micro-osmotic pumps containing gliquidone into the abdominal cavities of Goto-Kakizaki (GK) rats with diabetic nephropathy. Blood glucose, $24 \mathrm{~h}$ urinary protein, and $24 \mathrm{~h}$ urinary albumin levels were measured weekly. After 4 weeks of gliquidone therapy, pathological changes in the glomerular basement membrane (GBM) were examined using an electron microscope. Real-time PCR, western blotting, and immunohistochemistry were employed to detect glomerular expression of receptors for advanced glycation end products (RAGE) (AGER), protein kinase $C \beta(P K C \beta)$, and protein kinase $A$ (PKA) as well as tubular expression of the albumin reabsorption-associated proteins: megalin and cubilin. Human proximal tubular epithelial cells (HK-2 cells) were used to analyze the effects of gliquidone and advanced glycation end products (AGEs) on the expression of megalin and cubilin and on the absorption of albumin. Gliquidone lowered blood glucose, $24 \mathrm{~h}$ urinary protein, and $24 \mathrm{~h}$ urinary albumin levels in GK rats with diabetic nephropathy. The level of plasma C-peptide increased markedly and GBM and podocyte lesions improved dramatically after gliquidone treatment. Glomerular expression of RAGE and PKC $\beta$ decreased after gliquidone treatment, while PKA expression increased. AGEs markedly suppressed the expression of megalin and cubulin and the absorption of albumin in $\mathrm{HK}-2$ cells in vitro, whereas the expression of megalin and cubilin and the absorption of albumin were all increased in these cells after gliquidone treatment. In conclusion, gliquidone treatment effectively reduced urinary protein in GK rats with diabetic nephropathy by improving glomerular lesions and promoting tubular reabsorption.
\end{abstract}

\section{Key Words}

- gliquidone

- diabetic nephropathy

- proteinuria

- mechanism

\section{Introduction}

Diabetic nephropathy is one of the most common, chronic, complications of diabetes mellitus (DM) and is a major contributing factor in end-stage kidney pathology. The major pathogenic mechanism of diabetic nephropathy is the occurrence of glomerular microvascular lesions, though the underlying mechanism http://joe.endocrinology-journals.org DOI: 10.1530/JOE-13-0199
C 2014 Society for Endocrinology Printed in Great Britain
Journal of Endocrinology (2014) 220, 129-141 
responsible for the development of these lesions remains elusive.

Advanced glycation end products (AGEs) have been found to be a major cause of diabetic microvascular lesions. AGEs may act upon the glomerular capillary receptor of AGE (RAGE), activate protein kinase C (PKC), initiate oxidation stress reactions and/or cause a large accumulation of - reactive oxygen species (ROS) and nitric oxide, all of which can injure the glomerular microvascular endothelial cells (Brownlee 2001, Kanwar et al. 2008). AGEs can also increase extracellular matrix and mesangial cell proliferation (Ziyadeh 1993). The diabetic glomerular lesions include structural and functional changes in glomerular filtration membrane barriers, such as thickening of the glomerular basement membrane (GBM) and loss of podocytes (Bangstad et al. 1994, Wolf et al. 2005), resulting in increased protein excretion. In fact, tubular lesions have been found in diabetics even when the urinary albumin excretion rate is within a normal range. It has been reported that tubular lesions and renal interstitial fibrosis are more closely correlated to renal function than is glomerular sclerosis (Magri \& Fava 2009). AGE conjugates with RAGE to activate PKC and increases the production of cytokines (Kanwar et al. 2008). They also alter the expression of the reabsorptionassociated proteins, megalin and cubilin, on the membrane surfaces of epithelial cells and affect the reabsorption of urinary protein (Tojo et al. 2003, Amsellem et al. 2010). Glomerular vascular lesions lead to increased protein leakage and tubular lesions result in decreased protein reabsorption, therefore these lesions are the major pathophysiological cause for the occurrence of proteinuria in diabetic nephropathy (Tryggvason et al. 2006, Russo et al. 2009).

PKC activation in glomerular capillaries has been considered to be the most important link during the pathogenesis of diabetic nephropathy, and there is experimental evidence that PKC inhibitors could effectively prevent and control diabetic nephropathy (Tuttle et al. 2005). Recently, a correlation between the pathogenesis of diabetic nephropathy and decreased protein kinase A (PKA) activity in the glomerular capillary was identified. PKC activity is increased and PKA activity is decreased in diabetic nephropathy, thus the PKC/PKA balance is disturbed. Treatment with a PKA agonist may also effectively prevent and/or treat diabetic nephropathy (Wang et al. 2012).

Gliquidone is a second-generation hypoglycemic sulfonylurea that promotes the release of endogenous insulin from pancreatic $\beta$-cells and effectively lowers blood glucose. It is rapidly absorbed by the intestinal tract when taken orally and has a biphasic stimulatory effect on insulin secretion. Approximately $95 \%$ of gliquidone is excreted through the bile and feces so drug accumulation is not a problem in patients with renal insufficiency. Therefore, the World Health Organization has recommended it as a first-line drug for the treatment of mild to moderate renal lesions in patients with diabetic nephropathy. In addition with lowering the level of plasma glucose, gliquidone could boots the sensitivities of peripheral tissues and hepatocytes to insulin (Malaisse 2006) and enhances the transcription activities of peroxisome proliferator-activated receptor $\gamma$ (PPAR $\gamma$; Lee et al. 2011). Yarat et al. (2001) demonstrated that gliquidone markedly lowered the levels of nonenzymatic glycosylation protein and total protein and increased the level of lens glutathione in diabetic rats. Yanardag et al. (2005) reported that gliquidone lessened the STZ-induced hepatic lesions by reducing oxidative stress in diabetic rats. In patients with diabetic nephropathy, substitution of oral glibenclamide with gliquidone has increased the glomerular filtration rate and lowered urinary albumin (Mazurov et al. 1998).

Although some studies have demonstrated the effectiveness of gliquidone in preventing and controlling diabetic nephropathy, the mechanism by which it improves diabetic nephropathy remains elusive. It could be that a higher plasma drug concentration enhances the effect of gliquidone or that gliquidone improves diabetic nephropathy by directly acting on the kidney, in addition to lowering glycemic levels. In this present study, a microosmotic pump that released gliquidone at a constant rate was used to treat diabetic nephropathy in Goto-Kakizaki (GK) rats. Pathological and functional changes in the kidneys were examined, both before and after treatment, and the effects of gliquidone on glomerular and tubular phenotype and function were analyzed in order to further elucidate the molecular mechanism by which gliquidone improves diabetic nephropathy.

\section{Materials and methods}

\section{Animal model and groups}

SPF-grade 8-week-old male GK rats, weighing about 250-300 g, were purchased from Shanghai Slac Laboratory Animal, Inc. (Shanghai, China). Rats were maintained on a $12 \mathrm{~h}$ light: $12 \mathrm{~h}$ darkness cycle with free access to rodent chow and water. All animal experiments were performed in accordance with the protocols and guidelines approved

Published by Bioscientifica Ltd 
by the Animal Ethics Committee of China-Japan Friendship Hospital. After consumption of a high-fat diet for 2 weeks, blood glucose levels were determined in GK rats; rats that had blood glucose levels over $16.7 \mathrm{mM}$ for 3 consecutive days were classified as diabetic. Diabetic rats were then fed conventional chow for an additional 10 weeks and $24 \mathrm{~h}$ urinary albumin was determined. When the $24 \mathrm{~h}$ urinary albumin level was $>400 \mu \mathrm{g}$, the establishment of a diabetic nephropathy animal model was confirmed (Wang et al. 2012). A total of 18 GK rats with diabetic nephropathy were divided into three groups, according to a random number table $(n=6$ in each group), as follows. The gliquidone treatment group: an ALZET micro-amount osmotic pump, infused with $2 \mathrm{ml}$ gliquidone solution, was surgically implanted into the abdominal cavity. The gliquidone solution was formulated with $99.8 \%$ dimethylformamide and the gliquidone solution was released at a constant rate of $40 \mathrm{nmol} / \mathrm{kg}$ per min. The insulin treatment group: insulin glargine $2.5 \mathrm{IU}$ was administered once a day via s.c. injection. Diabetic nephropathy group: a micro-amount osmotic pump, infused with $2 \mathrm{ml}$ of $99.8 \%$ dimethylformamide, was implanted into the abdominal cavity and the solution was released at the same rate as in the gliquidone treatment group. Six SPF-grade, male Wistar rats of similar age and body weight (250-300 g) were used as controls. The treatment duration was 4 weeks in all groups.

\section{Detection of gliquidone concentration in the blood}

To evaluate the effectiveness of drug delivery via micro-osmotic pump, gliquidone (10 and $50 \mathrm{mg} / \mathrm{kg}$ body weight per day) was administrated to rats through the micro-osmotic pump or by intragastric administration. HPLC with fluorescence detection was used for determination of gliquidone in rat serum. Stable drug concentrations were detected in the blood of all groups after 1 week of drug administration.

\section{Detection of blood glucose, urinary protein, and urinary albumin}

Blood glucose, urinary protein, and urinary albumin levels were detected at weeks $0,1,2,3$, and 4 . Blood glucose levels were detected, via the tail vein, using a blood glucose meter (Johnson \& Johnson, New Brunswick, NJ, USA). Animals were kept in metabolic cages for collection of $24 \mathrm{~h}$ urine samples. A BCA protein kit (Beyotime, NanTong, China) and rat albumin ELISA kit (Assaypro,
St Charles, MO, USA) were used to measure urinary protein and urinary albumin levels.

\section{Detection of serum C-peptide}

After 4 weeks of treatment, animals were killed with 2.5\% pentobarbital sodium (i.p.) and serum was isolated from venous blood. A rat C-peptide RIA kit (R\&D, Shanghai, China) was used to measure the concentration of C-peptide.

\section{Electron microscopy}

Rat renal tissues were fixed in $2.5 \%$ glutaraldehyde for $30 \mathrm{~min}$ at room temperature, rinsed with PBS and postfixed with $1 \%$ osmic acid. The specimens were then dehydrated and embedded into Epon812 to prepare slides for electron microscopy. GBM and podocyte morphologies were observed using a JEM-1010 transmission electron microscope (JEOL, Tokyo, Japan).

\section{Isolation of glomerular and tubular tissues}

After the 4-week treatment, both kidneys were surgically harvested from each rat after killing. The renal cortex was homogenized and passed through a 100-mesh sieve. The filtrate was applied to a 300-mesh metal sieve and the remaining glomeruli and filter tubules were harvested for subsequent experiments.

\section{Human proximal tubular epithelial cell (HK-2 cell) culture}

Human proximal tubular epithelial cells (HK-2) were maintained in DMEM/F12 culture medium (Sigma) containing $10 \%$ inactivated fetal bovine serum, $100 \mathrm{U} / \mathrm{ml}$ penicillin, and $100 \mathrm{U} / \mathrm{ml}$ streptomycin. Digestion was done using $0.1 \%$ trypsin/0.1\% EDTA.

HK-2 cells were cultured in medium containing AGEs ( $400 \mu \mathrm{g} / \mathrm{ml}$, Abcam, Cambridge, UK), AGEs $(400 \mu \mathrm{g} / \mathrm{ml})$ + insulin $(1 \mathrm{IU} / \mathrm{ml})$, AGEs $(400 \mu \mathrm{g} / \mathrm{ml})+$ gliquidone $(1.0 \mu \mathrm{g} / \mathrm{ml}$, Double-crane Pharmaceuticals, Beijing, China), AGEs $(400 \mu \mathrm{g} / \mathrm{ml})+\mathrm{LY} 33531(10 \mathrm{nM})$ or AGEs $(400 \mu \mathrm{g} / \mathrm{ml})+8$-Br-cAMP $(100 \mu \mathrm{M})$ for either 24 (for realtime PCR) or $48 \mathrm{~h}$ (for albumin absorption and western blot). Albumin absorption and cubilin and megalin expression were evaluated as described below.

\section{Evaluation of albumin absorption using HK-2 cells}

The HK-2 cells were inoculated into a 96-well plate and cultured with AGEs, AGEs + insulin, AGEs + gliquidone,

Published by Bioscientifica Ltd 
AGEs + LY33531 or AGEs + 8-Br-cAMP for $48 \mathrm{~h}$. FITClabeled human albumin (final concentration of $20 \mu \mathrm{g} / \mathrm{ml}$, FITC-albumin, Abcam) was then added to the culture medium (Sidaway et al. 2004). After $30 \mathrm{~min}$, the culture medium was removed and the cells were washed four times with PBS. The cellular fluorescence intensity of each well was then detected using a continuous spectrum fluorescence plate reader (Molecular Devices, Sunnyvale, CA, USA). The protein concentration was analyzed by the BCA protein assay kit (Beyotime). The fluorescence intensity was corrected for cell numbers by total protein and expressed as fluorescence intensity per microgram of protein.

\section{Real-time PCR}

Total RNA was extracted using the SV total RNA isolation system (Promega) and $2 \mu \mathrm{g}$ of total RNA was reverse transcripted using a reverse transcription kit (Promega). The SYBR green real-time PCR Master Mix (Toyobo, Shanghai, China) and ABI 7500 sequence detection system (Applied Biosystems) were employed for PCR amplification using the three-step method. Relative quantification of transcript levels was performed by the $2^{-\Delta \Delta C t}$ method. The primers used for PCR amplification are shown in Supplementary Table 1, see section on supplementary data given at the end of this article (rat genes) and- Supplementary Table 2 (human genes).

\section{Western blot}

SDS-PAGE was used to fractionate $100 \mu \mathrm{g}$ total protein and the protein was then transferred onto a PVDF membrane. Immunoblotting was performed using mouse anti-actin antibody (Sigma), mouse anti-RAGE antibody (Sigma), mouse anti-rat/human PKC $\beta$ antibody (Sigma), rabbit anti-mouse/human PKA antibody (Abcam), murine anti-rat megalin antibody (Abcam), goat anti-rat cubilin antibody (Santa Cruz Biotechnology, Inc.), rabbit anti-human megalin antibody (Abcam), and goat anti-human cubilin antibody (Santa Cruz Biotechnology, Inc.) respectively. ECL (Millipore, Shanghai, China) was used for detection.

\section{Immunohistochemical staining}

Rat pancreas tissue was fixed in 10\% neutral formaldehyde and paraffin-embedded slides were prepared in order to evaluate insulin levels in the diabetic and gliquidone groups. The sections were immunostained with rabbit anti-rat insulin antibody and HRP-labeled secondary antibody. After undergoing a reaction with DAB substrate and re-staining the nuclei, the slides were observed and photographed under a light microscope (Olympus, Tokyo, Japan).

Rat kidneys were fixed in 10\% neutral formaldehyde and paraffin-embedded slides were prepared to evaluate the effect of gliquidone on protein reabsorption. The sections were immunostained with mouse anti-rat RAGE antibody, mouse anti-rat PKC $\beta$ antibody, rabbit anti-rat PKA antibody, mouse anti-rat megalin antibody, and goat anti-mouse cubilin antibody. Sections were then rinsed with PBS and HRP-labeled secondary antibody was added. After undergoing a reaction with DAB substrate and re-staining the nuclei, the slides were observed and photographed under a light microscope (Olympus).

\section{Immunofluorescent staining of cells}

HK-2 cells were inoculated into a 96-well culture plate and treated with AGEs $(400 \mu \mathrm{g} / \mathrm{ml})$ or AGEs $(400 \mu \mathrm{g} / \mathrm{ml})+$ gliquidone $(1 \mu \mathrm{g} / \mathrm{ml})$ for $48 \mathrm{~h}$. The cells were then washed with PBS, fixed with 4\% paraformaldehyde and blocked with $1 \%$ BSA/0.02\% Tween. Immunostaining was done using mouse anti-human RAGE antibody, mouse anti-human PKC $\beta$ antibody, rabbit anti-human PKA antibody, rabbit anti-human megalin antibody, and goat anti-human cubilin antibody as the primary antibodies and Alexa fluor 488-conjungated secondary antibodies. The results were observed and photographed using a fluorescence microscope.

\section{Statistical analyses}

The data are expressed as mean \pm s.D. The software SPSS 17.0 was used for statistical analyses. A one-way ANOVA was used for intergroup data comparison. $P<0.05$ and $P<0.01$ denote statistical significance.

\section{Results}

\section{Blood concentration of gliquidone after administration by micro-osmotic pump}

One week after treatment, the average serum concentration of gliquidone in the group treated via pump administration was approximately five times higher than in the group treated via intragastric administration. This was true for both the 10 and $50 \mathrm{mg} / \mathrm{kg}$ groups. Furthermore, the gliquidone serum concentration was five times higher in the group that received $50 \mathrm{mg} / \mathrm{kg}$

Published by Bioscientifica Ltd 


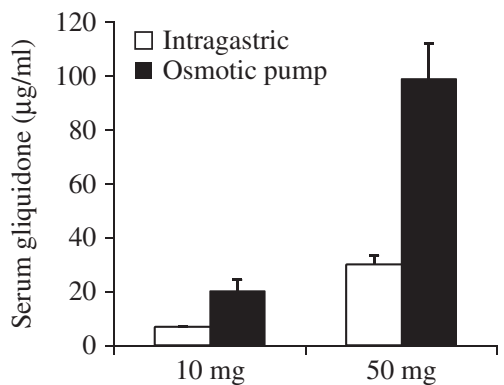

Figure 1

Concentration of gliquidone in the blood after administration by micro-osmotic pump. At the end of 1 week of treatment with gliquidone, serum was collected and the gliquidone concentration determined using HPLC with fluorescence detection. Intragastric: gliquidone was administrated intragastrically to GK rats once daily at a dose similar to that received by rats in the micro-osmotic pump group. Osmotic pump: gliquidone was administered to GK rats via a micro-osmotic pump that was implanted into the abdominal cavity. $10 \mathrm{mg}$ : drug dose is $10 \mathrm{mg} / \mathrm{kg}$ body weight; $50 \mathrm{mg}$ : drug dose is $50 \mathrm{mg} / \mathrm{kg}$ body weight.

compared with the group that received $10 \mathrm{mg} / \mathrm{kg}$ gliquidone. Thus, a higher blood concentration of gliquidone was obtained via i.p. administration using a microosmotic pump (Fig. 1).

\section{Effects of gliquidone on glycemia and proteinuria}

Blood glucose levels decreased markedly during the treatment period in both the gliquidone and insulin groups (Fig. 2A). The $24 \mathrm{~h}$ urinary protein and urinary albumin levels increased in the diabetic nephropathy and insulin groups. However, in the gliquidone group, the $24 \mathrm{~h}$ urinary protein level (Fig. 2B) did not increase and the $24 \mathrm{~h}$ urinary albumin level (Fig. 2C) increased at a slower rate. After 2 weeks of treatment, there were significant differences in the $24 \mathrm{~h}$ urinary protein and albumin levels between the diabetic nephropathy and gliquidone groups $(P<0.01)$.

\section{Effects of gliquidone on the secretion of insulin and C-peptide in rat islet cells}

Insulin staining decreased markedly in the diabetic nephropathy group compared with the normal group. However, insulin staining in the gliquidone group was increased and approximated the normal level (Fig. 3A). Furthermore, the serum concentration of C-peptide was markedly lower in the diabetic nephropathy group than in the control group, but markedly higher in the gliquidone group than in the diabetic nephropathy group (Fig. 3B).

\section{Effects of gliquidone on the morphologies of GBM and podocytes}

The GBM in GK rats with diabetic nephropathy was obviously thickened and the foot processes of the podocytes were condensed or missing and in disarray compared with those of the normal controls. After 4 weeks of treatment with gliquidone, the GBM was markedly thinner and the morphologies and arrays of podocyte foot processes improved and were similar to those of
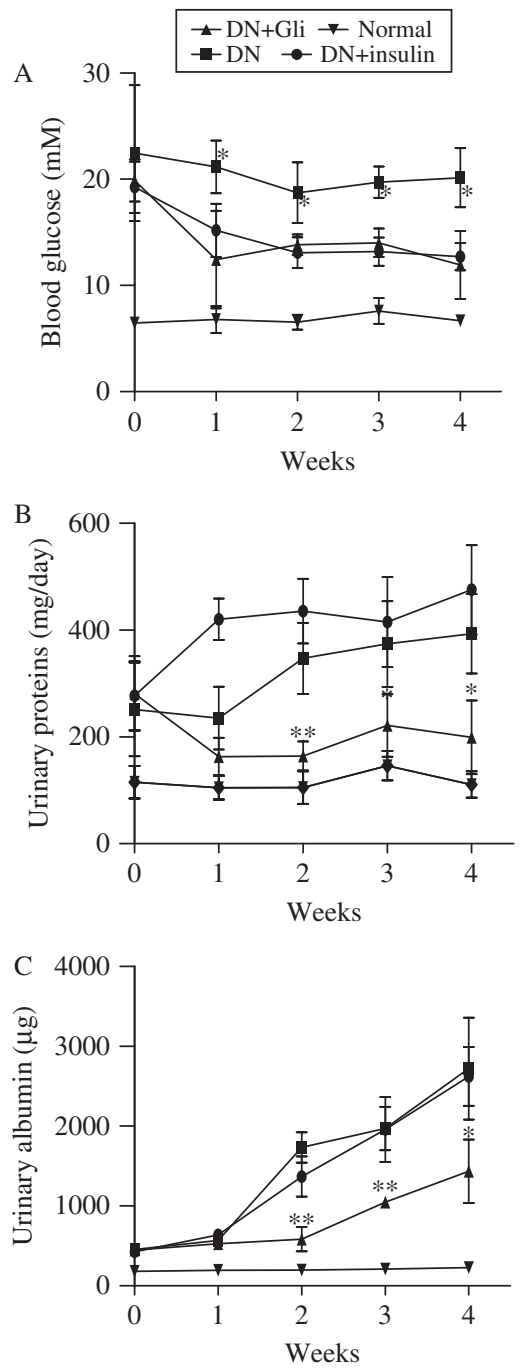

Figure 2

The effects of gliquidone on urinary albumin excretion rate in diabetic GK rats. (A) Effects of gliquidone on glycemic levels in diabetic GK rats. (B) Effects of gliquidone on urinary protein levels in diabetic GK rats. (C) Effects of gliquidone on urinary albumin levels in diabetic GK rats. Normal, normal rats; DN, diabetic nephropathy GK rats treated with saline; DN + Gli, diabetic nephropathy GK rats treated with gliquidone; $\mathrm{DN}+$ insulin, diabetic nephropathy GK rats treated with insulin. ${ }^{*} P<0.05$, $* * P<0.01 \mathrm{DN}+$ Gli vs DN group.

Published by Bioscientifica Ltd. 

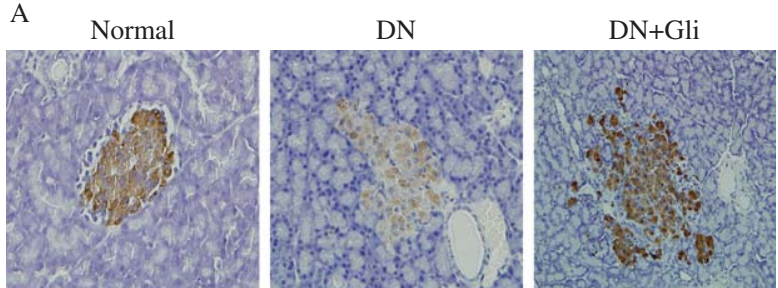

B

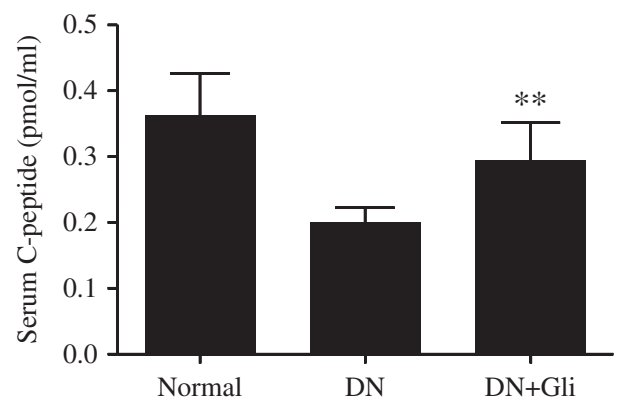

Figure 3

The effects of gliquidone on insulin expression in islets and C-peptide levels in serum. (A) The effect of gliquidone on insulin expression in islets. (B) The effect of gliquidone on serum C-peptide levels in diabetic rats. Normal, normal rats; DN, diabetic nephropathy GK rats treated with saline; DN + Gli, diabetic nephropathy GK rats treated with gliquidone. $* * P<0.01$ vs $D N$ group.

the normal group. However, after 4 weeks of insulin treatment, the morphologies of the GBM and foot processes were not obviously different from those of the diabetic nephropathy group (Fig. 4).

\section{Effects of gliquidone on the absorption of albumin by HK-2 cells}

Cultured HK-2 cells can absorb FITC-labeled human albumin from culture medium. After co-incubating with AGEs, the HK-2 cells exhibited a decreased intracellular fluorescence intensity compared with the control group. In the gliquidone group, the fluorescence intensity was stronger than in the AGEs group (Fig. 5A and B). Gliquidone increased the cellular absorption of albumin in a dose-dependent manner (Fig. 5C).

\section{Effects of gliquidone on the expression of megalin and cubilin in kidney tubules and HK-2 cells}

The expression of megalin and cubilin in the tubular tissue was decreased in the diabetic nephropathy group compared with the control group (Fig. 6A, B, and C). After 4 weeks of treatment with gliquidone, the tubular expression of megalin and cubilin was upregulated.
The expressions of megalin and cubilin were decreased in AGE-treated HK-2 cells compared with normal controls. However, gliquidone appeared to block the effects of AGE, as demonstrated by the normal expression levels of megalin and cubilin in the $\mathrm{AGE}+$ gliquidone group (Fig. 6D, E, and F).

\section{Effects of gliquidone on the glomerular expression of RAGE, PKC $\beta$, and PKA}

The glomerular expression of RAGE and PKC $\beta$ in GK rats with diabetic nephropathy was upregulated, while the expression of PKA was markedly decreased compared with controls. The expression of RAGE and PKC $\beta$ was decreased and the expression of PKA was increased in the gliquidone group compared with the diabetic nephropathy group (Fig. 7A, B, and C).

\section{Effects of gliquidone on the expression of RAGE, PKC $\beta$, and PKA in kidney tubules and HK-2 cells}

The changes in RAGE, PKC $\beta$, and PKA levels in tubular tissues of the three groups were similar to those found in the glomerulus of the three groups. The tubular expression of RAGE and PKC $\beta$ in GK rats with diabetic nephropathy GK rats was increased, while PKA expression was markedly decreased, compared with normal controls, at the mRNA, protein and tissue staining levels (Fig. 8A, B, and C). In the

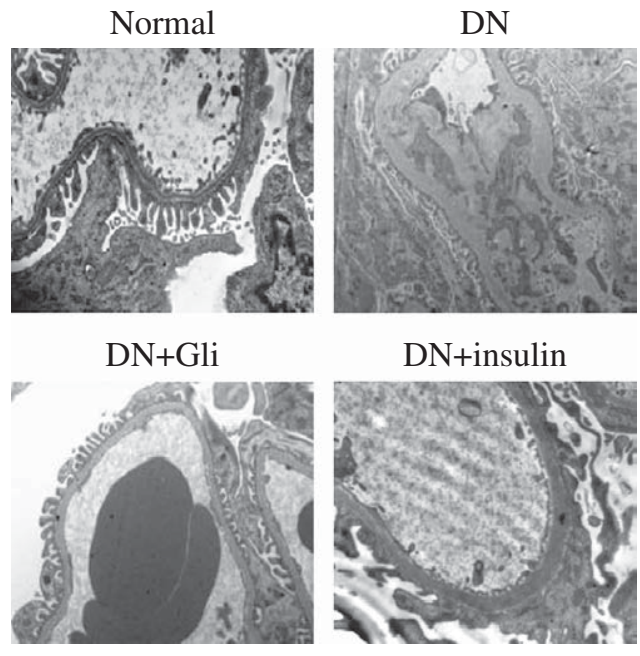

\section{Figure 4}

The effects of gliquidone on the structure of the GBM and on foot processes of podocytes. Normal, normal rats; DN, diabetic nephropathy GK rats treated with saline; DN + Gli, diabetic nephropathy GK rats treated with gliquidone; DN + insulin, diabetic nephropathy GK rats treated with insulin.

Published by Bioscientifica Ltd. 
A
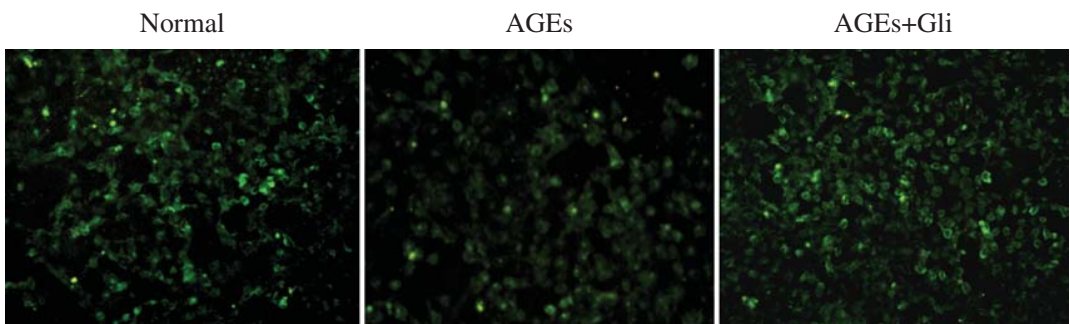

B

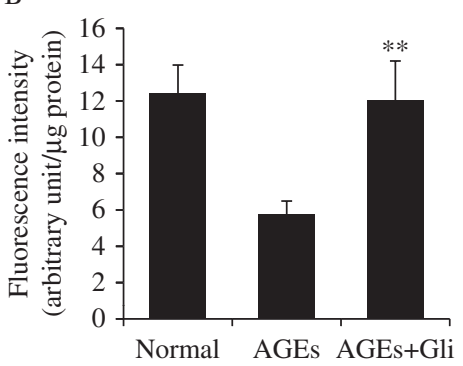

C

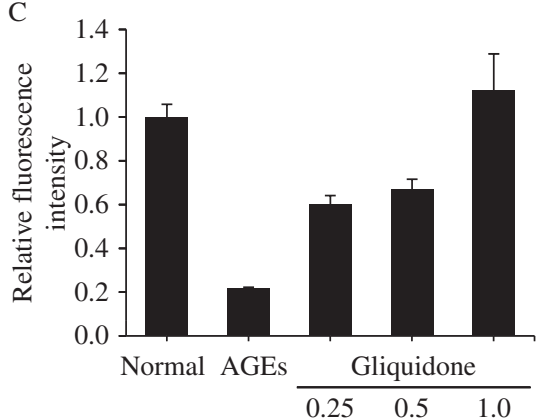

Figure 5

Effects of gliquidone on the absorption of albumin by HK-2 cells.

(A) HK-2 cells absorbed FITC-labeled human albumin as demonstrated by microscopy. (B) The quantitative analysis of absorption as measurements of fluorescence intensity under a fluorescence plate reader and corrected

gliquidone group, the tubular expression of RAGE and PKC $\beta$ was lower and PKA expression was higher compared with the diabetic nephropathy group.

In HK-2 cells, the expression of RAGE and PKC $\beta$ in the AGEs group was increased, while the expression of PKA was decreased compared with the normal control group. In the AGEs + gliquidone group, the expression of RAGE and PKC $\beta$ was downregulated and the expression of PKA was upregulated compared with the AGEs group (Fig. 8D, E, and F).

\section{Effects of a PKC inhibitor and PKA activator on albumin absorption function and megalin and cubilin expression in HK-2 cells}

As AGEs and gliquidone affect albumin absorption, along with PKC and PKA expression, the PKC inhibitor, LY333531 and PKA activator, 8-Br-cAMP were used to investigate the effect of PKC and PKA on albumin absorption and the expression of megalin and cubilin in HK-2 cells. The results indicated that the decreased albumin absorption found in AGEs-treated HK-2 cells could be reversed by co-incubating the cells with gliquidone, LY333531 or 8-Br-cAMP, but not with insulin (Fig. 9A). The decreased expression of megalin and cubilin in AGEs-treated HK-2 cells was also reversed by cell numbers by total protein. (C) Dose-effect of gliquidone on albumin absorption. All of the experiments were performed in triplicate. Four wells were set up for each group. ${ }^{*} P<0.01$ vs AGEs group.

gliquidone, LY333531 and 8-Br-cAMP, but not by insulin (Fig. 9B and C).

\section{Discussion}

Diabetic nephropathy is one of the most serious complications of DM. However, the pathogenic mechanism of diabetic nephropathy has remained elusive and there is no specific, efficacious drug treatment for diabetic nephropathy. Therefore, finding an effective drug to treat diabetic nephropathy has been a shared goal of researchers in recent years.

In this study, we found that gliquidone markedly lowered protein levels in the urine and improved nephropathic lesions in GK rats with diabetic nephropathy. Improvement of diabetic nephropathy with gliquidone was closely correlated with improved glomerular filtration and tubular reabsorption of albumin. These results provide evidence supporting the use of gliquidone for the prevention and treatment of diabetic nephropathy.

DM-induced microvascular lesions are considered to be one of the major causes of diabetic nephropathy. When glucose metabolism is disturbed, the production of AGEs increases; the AGEs act on the glomerular capillary receptor (i.e., RAGE) and promote oxidative stress by

Published by Bioscientifica Ltd 

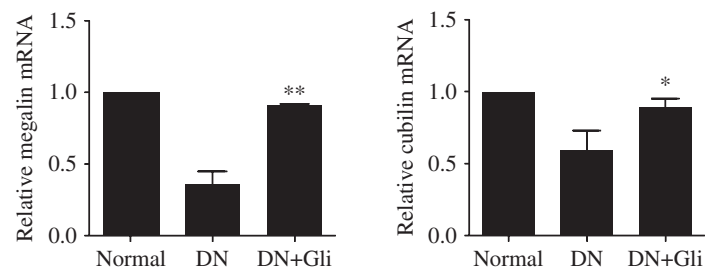

B

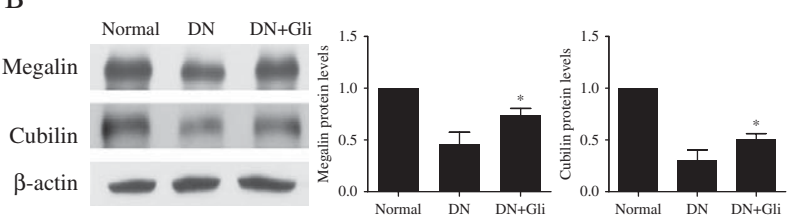

$\mathrm{C}$

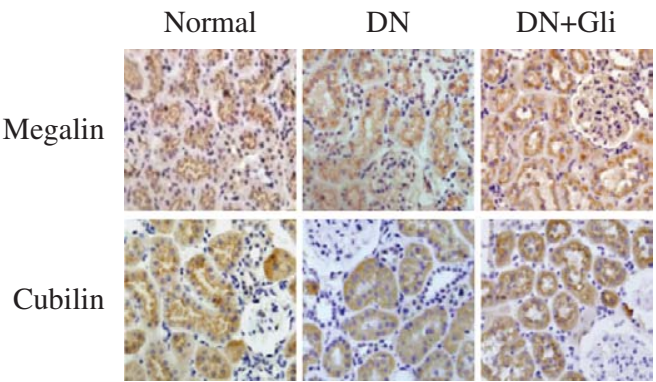

Figure 6

Effects of gliquidone on megalin and cubilin expression in kidney tubules of diabetic rats and in HK-2 cells. (A) The expression of megalin and cubilin in tubular tissue was evaluated using real-time PCR (A), western blotting (B), and immunohistochemical staining (C). The expression of

activating PKC, leading to an accumulation of ROS and NO that results in glomerular capillary endothelial cell injury. PKC has been reported to play a role in the pathogenesis of diabetic nephropathy, by contributing to cytokine activation, growth of extracellular matrix, cellular growth and proliferation, angiogenesis, vascular contraction and dilatation, and changes in vascular permeability (Noh \& King 2007, Geraldes \& King 2010). Within the PKC family, the isoform PKC $\beta$ has the greatest effect on the occurrence and development of diabetic nephropathy (Meier et al. 2009, Geraldes \& King 2010). The over-expression of PKC $\beta$ leads to upregulation of TGF- $\beta$ expression, which then induces the deposition of extracellular matrix, leading to glomerular sclerosis and tubular fibrosis (Koya et al. 1997, Slattery et al. 2008, Wu et al. 2009). Previous studies have demonstrated that the progression of diabetic nephropathy is delayed by knocking out the PKC $\beta$ (Prkcb) gene or by administering a PKC $\beta$ inhibitor (Tuttle et al. 2005, Meier et al. 2009). It is generally accepted that PKC activation promotes,
$\mathrm{D}$
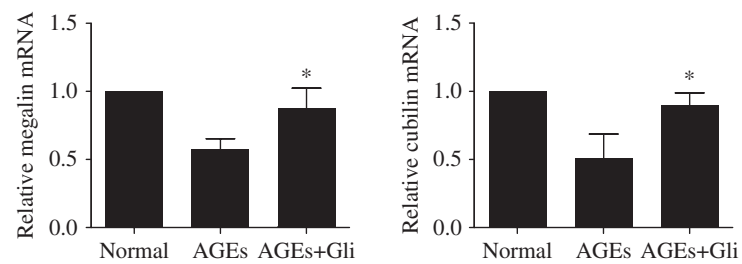

$\mathrm{E}$

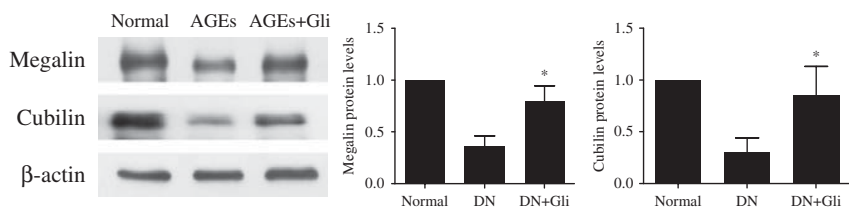

$\mathrm{F}$

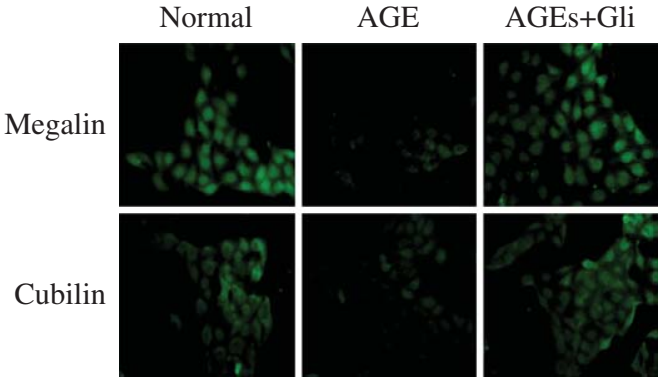

megalin and cubilin in HK-2 cells was evaluated using real-time PCR (D), western blotting $(E)$, and immunofluorescence staining $(F) .{ }^{\star} P<0.05$ $* * P<0.01$ vs DN group.

while PKC inhibition arrests, the occurrence of diabetic nephropathy (Noh \& King 2007). Recently, we have found that PKA activity is also closely correlated with the occurrence of diabetic nephropathy. The use of a PKA agonist can control or improve diabetic nephropathy (Wang et al. 2012).

In this present study, we found a marked decrease in microvascular lesions, in rats with diabetic nephropathy, after treatment with gliquidone. This was manifested by a downregulation in the expression of glomerular capillary RAGE, a reduction in PKC activity and an increase in PKA activity. In addition, the thickness of the GBM decreased and the morphology and distribution of podocytes improved. Biochemical analyses demonstrated a marked decline in both the $24 \mathrm{~h}$ urinary protein and $24 \mathrm{~h}$ urinary albumin levels, indicating that gliquidone improves glomerular filtration.

Islet transplantation has been reported to prevent or reverse diabetic microvascular lesions and to delay the onset of increased urinary protein in recipients

Published by Bioscientifica Ltd. 
A

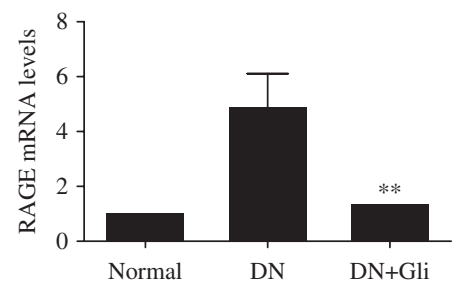

Normal DN DN+Gli

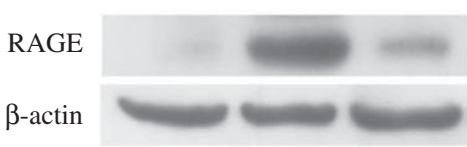

B

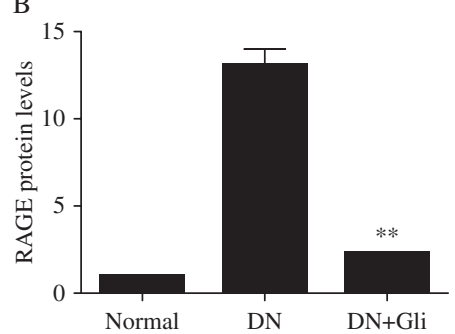

C

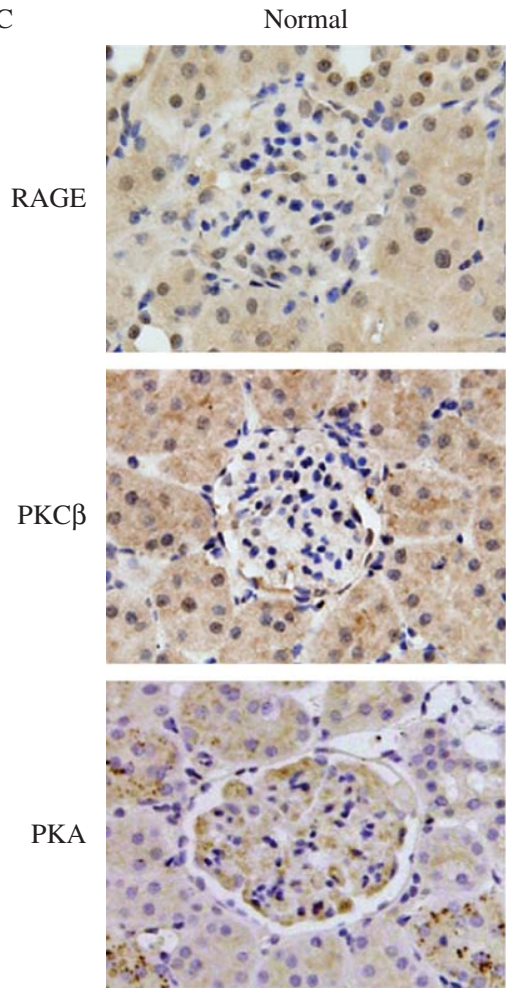

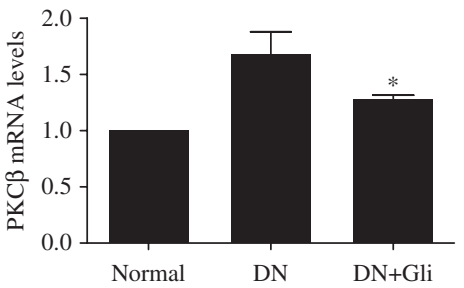
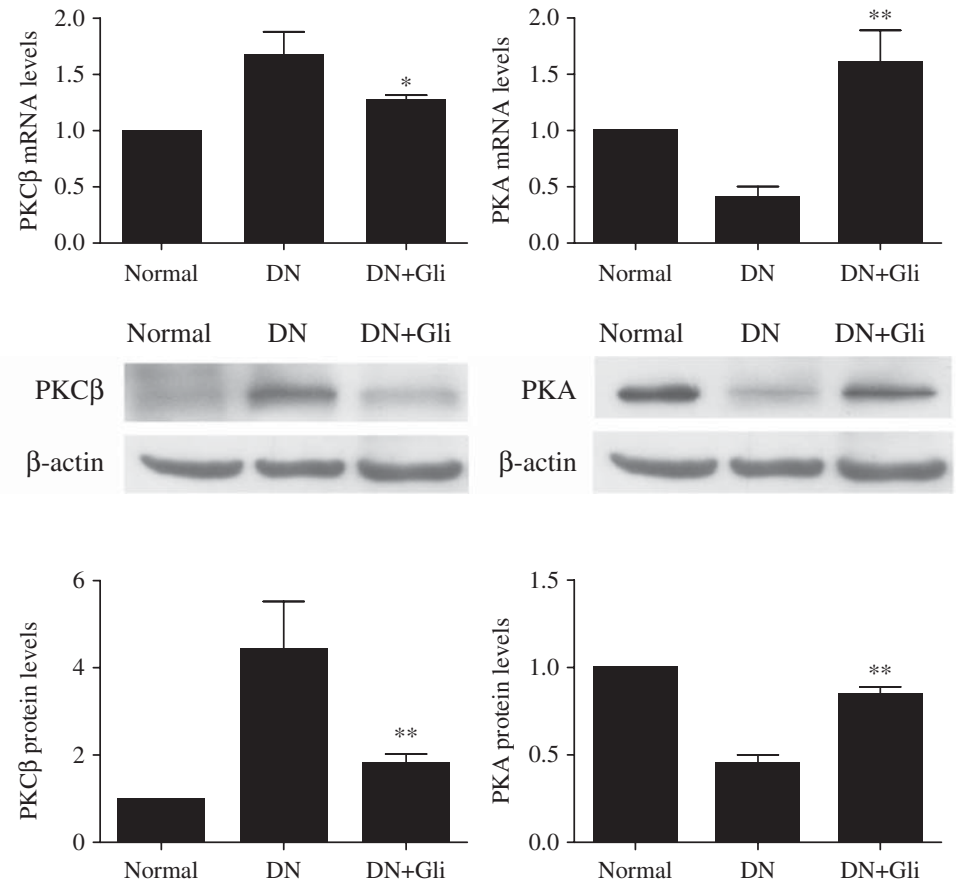

DN
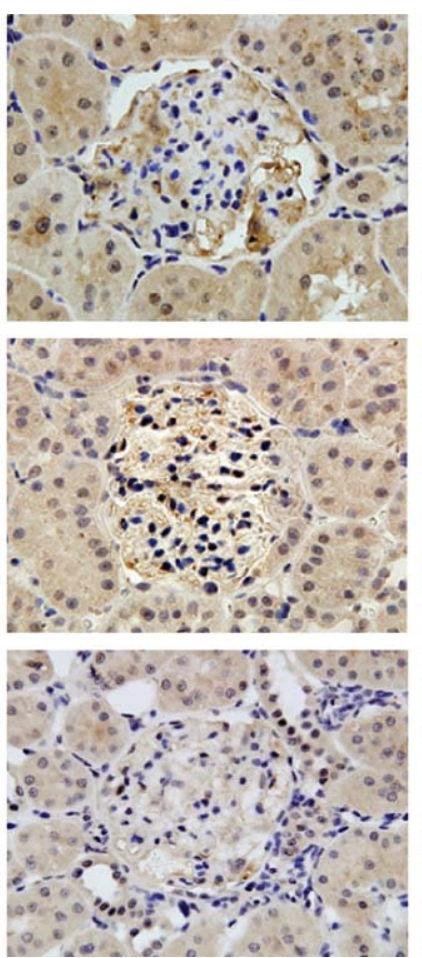
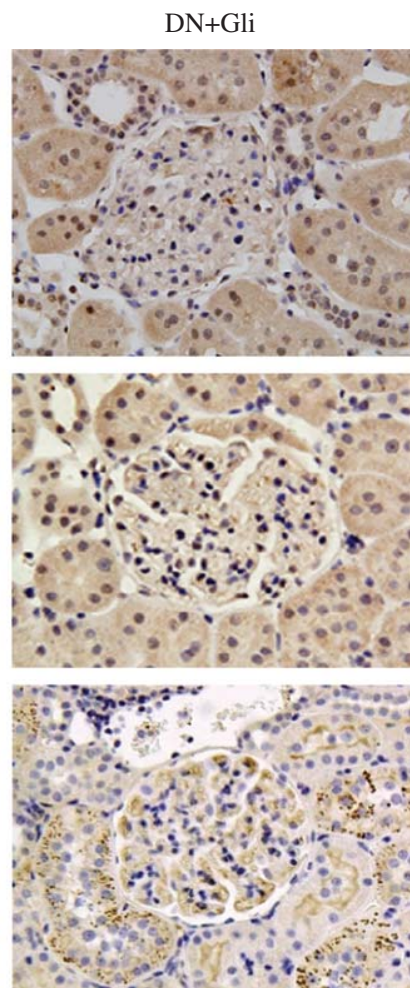

Figure 7

Effects of gliquidone on RAGE, PKC $\beta$, and PKA expression in the glomeruli of diabetic rats. Real-time PCR (A), western blotting (B), and immunohistochemical staining $(C)$ were used to evaluate the expression of megalin and cubilin in glomerular tissue. ${ }^{2} P<0.05, * \star P<0.01$ vs $D N$ group.

http://joe.endocrinology-journals.org DOI: 10.1530/JOE-13-0199
(C) 2014 Society for Endocrinology Printed in Great Britain
Published by Bioscientifica Ltd 

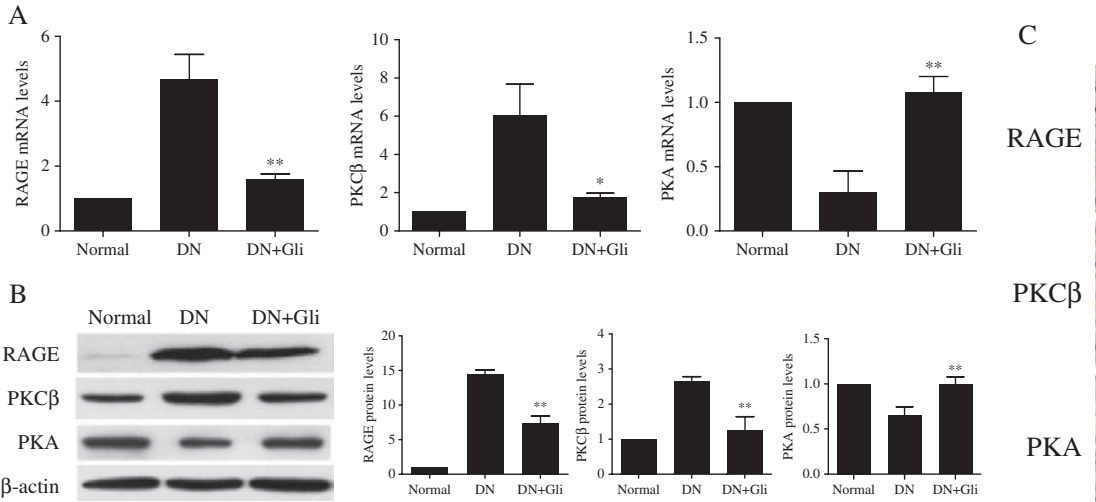

PKC $\beta$
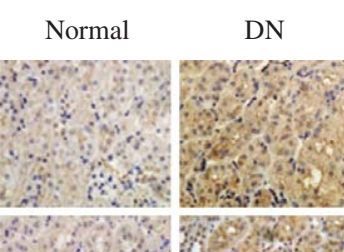

$\mathrm{DN}+\mathrm{Gli}$
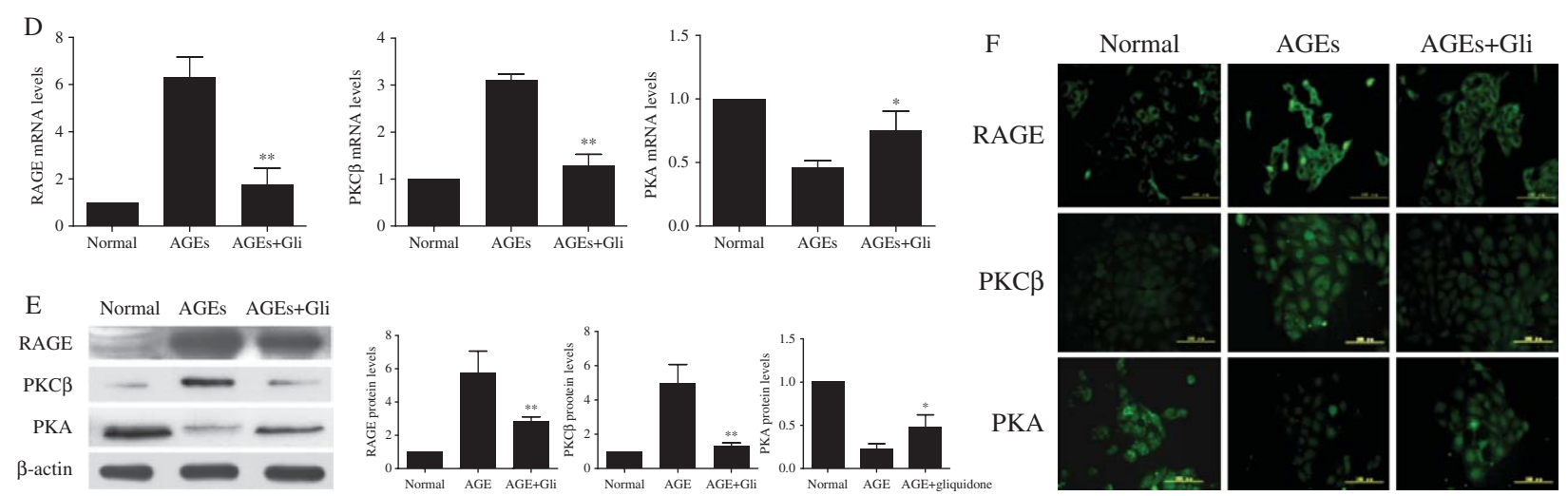

PKC $\beta$

PKA

\section{Figure 8}

Effects of gliquidone on RAGE, PKC $\beta$, and PKA expression in kidney tubules of diabetic rats and in HK-2 cells. The expression of RAGE, PKC $\beta$, and PKA in tubular tissue was evaluated using real-time PCR (A), western blotting (B) and immunohistochemical staining (C). The expression of RAGE, PKC $\beta$, and PKA in HK-2 cells were evaluated using real-time PCR (D), western blotting $(\mathrm{E})$, and immunofluorescence staining $(\mathrm{F}) .{ }^{\star} P<0.05, * * P<0.01$ vs AGEs group.
(Fiorina et al. 2003, 2005). Recent studies have indicated that C-peptide produced by islet cells might help to prevent and treat diabetic microvascular lesions (Cotter et al. 2003, Forst \& Kunt 2004, Hills et al. 2010). In fact, several human trials and animal studies have demonstrated that C-peptide can improve the kidney structural and functional abnormalities of diabetic nephropathy (Hills et al. 2010). Samnegard et al. (2005) reported a marked decrease in proteinuria and mesangial matrix synthesis in diabetic nephropathy rats treated with Cpeptide compared with those treated with placebo. In this present study, gliquidone was found to elevate the level of C-peptide in the plasma (Fig. 3B) of diabetic GK rats. This may be one of the ways that gliquidone improves diabetic neuropathy.

Proteinuria is one of the major clinical manifestations of diabetic nephropathy and is closely correlated with the degree of renal lesions (Araki et al. 2008). The structural alterations in glomerular filtration barriers and tubular reabsorption of protein are the major pathophysiological determinants for the occurrence of proteinuria in diabetic nephropathy. Changes in the GBM and podocytes damage the glomerular filtration barriers. The lesions in the GBM and podocytes clearly improved after gliquidone treatment and this is probably one of the reasons for the lower levels of urinary protein and albumin that were observed after treatment with gliquidone.

Reduced tubular reabsorption of proteins is another major contributor to proteinuria in diabetic nephropathy (Russo et al. 2009). It is known that tubular epithelial cells express RAGE on their surfaces, which conjugate with AGEs. AGEs can induce the occurrence of oxidative stress through RAGE by producing a large amount of ROS, activating the rennin-angiotensin system, interacting with signaling molecules such as PKC and nuclear factor kappa $\mathrm{B}(\mathrm{NF}-\kappa \mathrm{B})$ and causing tubular interstitial fibrosis 

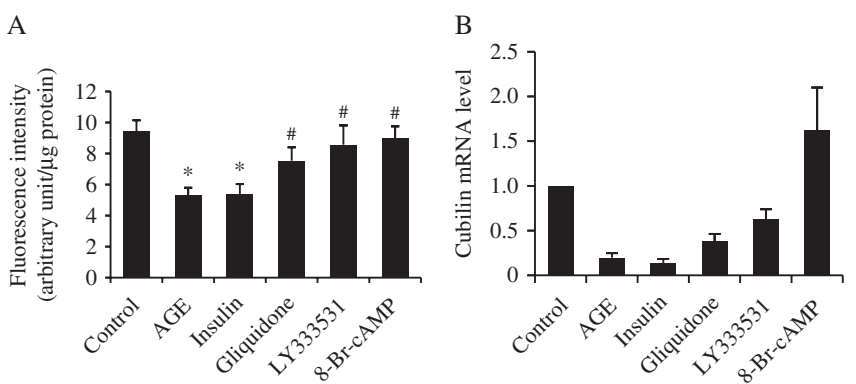
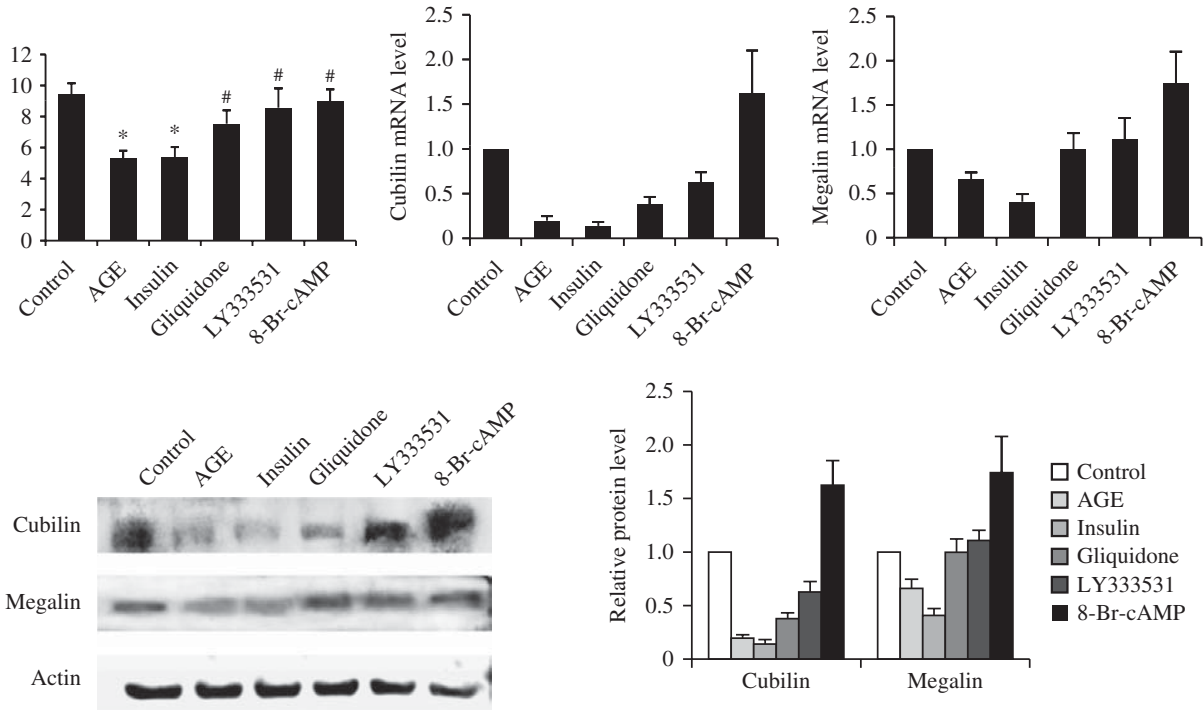

Figure 9

Effects of a PKC inhibitor and a PKA activator on albumin absorption function and megalin and cubilin expression in HK-2 cells. (A) The effects of LY333531 (PKC inhibitor) and 8-Br-CAMP (PKA activator) on albumin absorption by HK-2 cells. ${ }^{*} P<0.01$ vs control group; $\# P<0.01$ vs $A G E$ group.

(Yamagishi \& Matsui 2010). The proximal renal tubule is a major site of reabsorption and is particularly sensitive to DM-associated metabolites and activated factors. Therefore, changes in proximal tubular epithelial cells contribute to the occurrence of tubular lesions in diabetic nephropathy. In this study, the proximal tubular expression of RAGE was upregulated, PKC activity was increased and PKA activity was decreased in rats with diabetic nephropathy. The proximal tubular expression of RAGE was downregulated and the PKC and PKA activities were normalized in rats with diabetic nephropathy that were treated with gliquidone. Furthermore, we confirmed that AGEs were the major cause of phenotypic changes in HK-2 cells and found that gliquidone could reverse these changes.

The reabsorption of albumin in proximal tubular epithelial cells is accomplished through the effects of two receptor proteins, megalin and cubilin (Verroust et al. 2002). The altered expression of these proteins can affect the reabsorption of albumin (Hosojima et al. 2009, Cabezas et al. 2011). In the present study, the proximal tubular expression of megalin and cubilin were markedly downregulated in GK rats with diabetic nephropathy, however, the expression was normalized after treatment with gliquidone. These findings were further confirmed using HK-2 cells. Our results demonstrated that AGEs

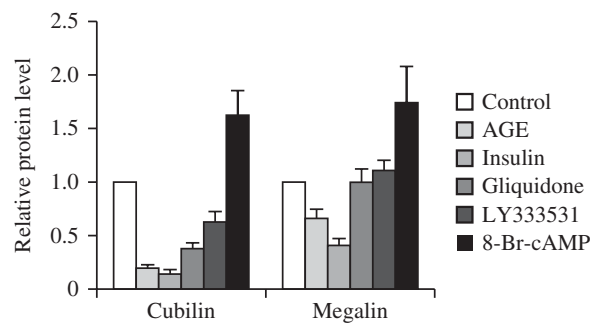

(B) The effects of LY333531 and 8-Br-CAMP on the expression of megalin and cubilin in HK-2 cells determined by real-time PCR. (C) The effects of LY333531 and 8-Br-CAMP the expression of megalin and cubilin in HK-2 cells determined by western blotting.

lowered albumin uptake by downregulating the expression of megalin and cubilin, while gliquidone increased albumin uptake in a dose-dependent manner by upregulating the expression of megalin and cubilin. This supports the idea that gliquidone lowers the urinary protein level in diabetic nephropathy by increasing the tubular reabsorption of albumin.

It should be stressed that administration of gliquidone via a micro-osmotic pump is probably different from oral administration in terms of therapeutic efficacy. A higher plasma concentration might be obtained with the use of a micro-osmotic pump. If this is the case, the renal excretion of gliquidone will be higher and the glomerular and tubular effects might be more apparent. Gliquidone is predominantly metabolized through the portal vein system so the plasma concentration is low when it is administered orally. Indeed, after oral intake, the renal excretion concentration is estimated to be only $5 \%$ of the total concentration. As a result, it may be difficult to control diabetic nephropathy and lower urinary protein through oral administration of gliquidone.

Our results indicate that gliquidone can effectively decrease urinary protein by improving glomerular lesions and promoting tubular reabsorption in GK rats with diabetic nephropathy. Gliquidone has the potential to be an effective therapeutic drug for the treatment of diabetic

Published by Bioscientifica Ltd. 
nephropathy, however pharmacokinetic differences between osmotic pump and oral administration must be taken into consideration.

\section{Supplementary data}

This is linked to the online version of the paper at http://dx.doi.org/10.1530/ JOE-13-0199.

\section{Declaration of interest}

The authors declare that there is no conflict of interest that could be perceived as prejudicing the impartiality of the research reported.

\section{Funding}

This study was supported by: the National Basic Research Program of China (2012CB966402); the Key New Drug Creation and Manufacture Program (No.2011ZX09102-010-03); and the National Nature Science Foundation of China (No. 81370873 and 81302334).

\section{References}

Amsellem S, Gburek J, Hamard G, Nielsen R, Willnow TE, Devuyst O, Nexo E, Verroust PJ, Christensen EI \& Kozyraki R 2010 Cubilin is essential for albumin reabsorption in the renal proximal tubule. Journal of the American Society of Nephrology 21 1859-1867. (doi:10.1681/ASN. 2010050492)

Araki S, Haneda M, Koya D, Kashiwagi A, Uzu T \& Kikkawa R 2008 Clinical impact of reducing microalbuminuria in patients with type 2 diabetes mellitus. Diabetes Research and Clinical Practice 82(Suppl 1) S54-S58. (doi:10.1016/j.diabres.2008.09.031)

Bangstad HJ, Dahl-Jørgensen K, Hanssen K, Østerby R, Berg K \& Hartmann A 1994 Improvement of blood glucose control in IDDM patients retards the progression of morphological changes in early diabetic nephropathy. Diabetologia 37 483-490. (doi:10.1007/s001250050136)

Brownlee M 2001 Biochemistry and molecular cell biology of diabetic complications. Nature 414 813-820. (doi:10.1038/414813a)

Cabezas F, Lagos J, Cespedes C, Vio CP, Bronfman M \& Marzolo MP 2011 Megalin/LRP2 expression is induced by peroxisome proliferatoractivated receptor- $\alpha$ and $-\gamma$ : implications for PPARs' roles in renal function. PLOS ONE 6 e16794. (doi:10.1371/journal.pone.0016794)

Cotter MA, Ekberg K, Wahren J \& Cameron NE 2003 Effects of proinsulin C-peptide in experimental diabetic neuropathy: vascular actions and modulation by nitric oxide synthase inhibition. Diabetes $\mathbf{5 2}$ 1812-1817. (doi:10.2337/diabetes.52.7.1812)

Fiorina P, Folli F, Bertuzzi F, Maffi P, Finzi G, Venturini M, Socci C, Davalli A, Orsenigo E, Monti L et al. 2003 Long-term beneficial effect of islet transplantation on diabetic macro-/microangiopathy in type 1 diabetic kidney-transplanted patients. Diabetes Care 26 1129-1136. (doi:10.2337/diacare.26.4.1129)

Fiorina P, Venturini M, Folli F, Losio C, Maffi P, Placidi C, La Rosa S, Orsenigo E, Socci C, Capella C et al. 2005 Natural history of kidney graft survival, hypertrophy, and vascular function in end-stage renal disease type 1 diabetic kidney-transplanted patients: beneficial impact of pancreas and successful islet cotransplantation. Diabetes Care $\mathbf{2 8}$ 1303-1310. (doi:10.2337/diacare.28.6.1303)

Forst T \& Kunt T 2004 Effects of C-peptide on microvascular blood flow and blood hemorheology. Experimental Diabesity Research 5 51-64. (doi:10.1080/15438600490424532)
Geraldes P \& King GL 2010 Activation of protein kinase C isoforms and its impact on diabetic complications. Circulation Research 106 1319-1331. (doi:10.1161/CIRCRESAHA.110.217117)

Hills CE, Brunskill NJ \& Squires PE 2010 C-peptide as a therapeutic tool in diabetic nephropathy. American Journal of Nephrology 31 389-397. (doi:10.1159/000289864)

Hosojima M, Sato H, Yamamoto K, Kaseda R, Soma T, Kobayashi A, Suzuki A, Kabasawa H, Takeyama A, Ikuyama K et al. 2009 Regulation of megalin expression in cultured proximal tubule cells by angiotensin II type $1 \mathrm{~A}$ receptor- and insulin-mediated signaling cross talk. Endocrinology 150 871-878. (doi:10.1210/en.2008-0886)

Kanwar YS, Wada J, Sun L, Xie P, Wallner EI, Chen S, Chugh S \& Danesh FR 2008 Diabetic nephropathy: mechanisms of renal disease progression. Experimental Biology and Medicine 233 4. (doi:10.3181/0705-MR-134)

Koya D, Jirousek MR, Lin YW, Ishii H, Kuboki K \& King GL 1997 Characterization of protein kinase $\mathrm{C} \beta$ isoform activation on the gene expression of transforming growth factor- $\beta$, extracellular matrix components, and prostanoids in the glomeruli of diabetic rats. Journal of Clinical Investigation 100 115-126. (doi:10.1172/ JCI119503)

Lee KW, Ku YH, Kim M, Ahn BY, Chung SS \& Park KS 2011 Effects of sulfonylureas on peroxisome proliferator-activated receptor $\gamma$ activity and on glucose uptake by thiazolidinediones. Diabetes \& Metabolism Journal 35 340-347. (doi:10.4093/dmj.2011.35.4.340)

Magri CJ \& Fava S 2009 The role of tubular injury in diabetic nephropathy. European Journal of Internal Medicine 20 551-555. (doi:10.1016/j.ejim. 2008.12.012)

Malaisse WJ 2006 Gliquidone contributes to improvement of type 2 diabetes mellitus management: a review of pharmacokinetic and clinical trial data. Drugs in $R \& D \quad 7$ 331-337. (doi:10.2165/00126839. 200607060-00002)

Mazurov VI, Novik AA, Nagibovich OA, Romashevskii BV, Guliaeva IV \& Kurganova TA 1998 Effect of sugar-reducing therapy on renal function in patients with type II diabetes mellitus. Klinicheskaia Meditsina 76 38-41.

Meier M, Menne J \& Haller H 2009 Targeting the protein kinase C family in the diabetic kidney: lessons from analysis of mutant mice. Diabetologia 52 765-775. (doi:10.1007/s00125-009-1278-y)

Noh H \& King GL 2007 The role of protein kinase C activation in diabetic nephropathy. Kidney International 72(Suppl) S49-S53. (doi:10.1038/sj.ki.5002386)

Russo LM, Sandoval RM, Campos SB, Molitoris BA, Comper WD \& Brown D 2009 Impaired tubular uptake explains albuminuria in early diabetic nephropathy. Journal of the American Society of Nephrology 20489. (doi:10.1681/ASN.2008050503)

Samnegard B, Jacobson SH, Jaremko G, Johansson BL, Ekberg K, Isaksson B, Eriksson L, Wahren J \& Sjoquist M 2005 C-peptide prevents glomerular hypertrophy and mesangial matrix expansion in diabetic rats. Nephrology, Dialysis, Transplantation 20 532-538. (doi:10.1093/ndt/ gfh683)

Sidaway JE, Davidson RG, McTaggart F, Orton TC, Scott RC, Smith GJ \& Brunskill NJ 2004 Inhibitors of 3-hydroxy-3-methylglutaryl-CoA reductase reduce receptor-mediated endocytosis in opossum kidney cells. Journal of the American Society of Nephrology 15 2258-2265. (doi:10.1097/01.ASN.0000138236.82706.EE)

Slattery C, Ryan MP \& McMorrow T 2008 Protein kinase C $\beta$ overexpression induces fibrotic effects in human proximal tubular epithelial cells. International Journal of Biochemistry \& Cell Biology 40 2218-2229. (doi:10.1016/j.biocel.2008.03.005)

Tojo A, Onozato ML, Kurihara H, Sakai T, Goto A \& Fujita T 2003 Angiotensin II blockade restores albumin reabsorption in the proximal tubules of diabetic rats. Hypertension Research 26 413-419. (doi:10.1291/ hypres.26.413)

Tryggvason K, Patrakka J \& Wartiovaara J 2006 Hereditary proteinuria syndromes and mechanisms of proteinuria. New England Journal of Medicine 354 1387-1401. (doi:10.1056/NEJMra052131) 
Tuttle KR, Bakris GL, Toto RD, McGill JB, Hu K \& Anderson PW 2005 The effect of ruboxistaurin on nephropathy in type 2 diabetes. Diabetes Care 28 2686-2690. (doi:10.2337/diacare.28.11.2686)

Verroust PJ, Birn H, Nielsen R, Kozyraki R \& Christensen EI 2002 The tandem endocytic receptors megalin and cubilin are important proteins in renal pathology. Kidney International 62 745-756. (doi:10.1046/j.1523-1755.2002.00501.x)

Wang H, Jiang YW, Zhang WJ, Xu SQ, Liu HL, Yang WY \& Lou JN 2012 Differential activations of PKC/PKA related to microvasculopathy in diabetic GK rats. American Journal of Physiology. Endocrinology and Metabolism 302 E173-E182. (doi:10.1152/ajpendo.00184.2011)

Wolf G, Chen S \& Ziyadeh FN 2005 From the periphery of the glomerular capillary wall toward the center of disease: podocyte injury comes of age in diabetic nephropathy. Diabetes 54 1626-1634. (doi:10.2337/ diabetes.54.6.1626)

Wu D, Peng F, Zhang B, Ingram AJ, Kelly DJ, Gilbert RE, Gao B \& Krepinsky JC 2009 PKC- $\beta 1$ mediates glucose-induced Akt activation and TGF- $\beta 1$ upregulation in mesangial cells. Journal of the American Society of Nephrology 20 554-566. (doi:10.1681/ASN. 2008040445)

Yamagishi S \& Matsui T 2010 Advanced glycation end products, oxidative stress and diabetic nephropathy. Oxidative Medicine and Cellular Longevity 3 101-108. (doi:10.4161/oxim. 3.2.11148)

Yanardag R, Ozsoy-Sacan O, Orak H \& Ozgey Y 2005 Protective effects of glurenorm (gliquidone) treatment on the liver injury of experimental diabetes. Drug and Chemical Toxicology 28 483-497. (doi:10.1080/01480540500262961)

Yarat A, Tunali T, Yanardag R, Gursoy FO, Sacan OO, Emekli N, Utuner A \& Ergenekon G 2001 The effect of Glurenorm (gliquidone) on lenses and skin in experimental diabetes. Free Radical Biology \& Medicine 31 1038-1042. (doi:10.1016/S0891-5849(01)00693-1)

Ziyadeh F 1993 The extracellular matrix in diabetic nephropathy. American Journal of Kidney Diseases 22 736-744.

Received in final form 12 October 2013

Accepted 15 October 2013

Accepted Preprint published online 19 October 2013
(C) 2014 Society for Endocrinology Printed in Great Britain
Published by Bioscientifica Ltd. 